\title{
UMBRAL CALCULUS, BINOMIAL ENUMERATION AND CHROMATIC POLYNOMIALS
}

\author{
NIGEL RAY
}

\begin{abstract}
We develop the concept of partition categories, in order to extend the Mullin-Rota theory of binomial enumeration, and simultaneously to provide a natural setting for recent applications of the Roman-Rota umbral calculus to computations in algebraic topology. As a further application, we describe a generalisation of the chromatic polynomial of a graph.
\end{abstract}

0. Introduction. In the last 20 years, the 19th century processes of the umbral calculus have undergone a radical transformation into modern and rigorous language at the hands of G.-C. Rota and his coworkers, culminating in the recent exposition of S. Roman [19].

One of the central influences in this development was the application by R. Mullin and Rota to the theory of binomial enumeration [13], subsequently reinterpreted by several authors, e.g. $[\mathbf{7}, \mathbf{9}]$. This theory was anticipated by Rota's seminal inquiries into the nature of Möbius functions on a poset [20], which were in turn inspired by ideas of $P$. Hall [6]. These concepts are still being extended into areas such as category theory [5], and are proving ever more fruitful in the originating environment of finite group theory, e.g. see [21].

Recently $[15,16]$, we have suggested that the modern umbral calculus also provides an enlightening setting for certain computations in algebraic topology, related to complex cobordism, generalised Bernoulli numbers and stable homotopy theory.

These disparate threads are rich in interconnections, but as yet there seems to be no obvious global viewpoint. Our aim here is to try and begin such a unification by proposing a combinatorial framework, applicable to each of the constituent theories, which descends directly from the philosophy of Mullin and Rota. We utilise the fundamental ideas of A. Joyal's theory of species [9], which has recently been extended to partitionals by O. Nava and Rota [14]. Some of our work is related to theirs, but differs substantially in detail and emphasis.

Our central theme is a purely combinatorial description of the associated and conjugate polynomial sequences of a $\Delta$-operator $e^{\alpha D}-1$ over a suitable ring $A$. This is achieved by introducing the concept of a partition category over $A$, which arises by enriching the structure of certain posets of partitions so as to create a category in which each arrow has a weight in $A$. The universal, or generic, example gives the weight of an arrow as the type of the defining partition.

We then extend the standard notions of incidence algebra, and zeta and Möbius functions, to the corresponding setting. It is in this context that we describe our

Received by the editors June 9, 1987.

1980 Mathematics Subject Classification (1985 Revision). Primary 06A10; Secondary 05C15. 
polynomial sequences. We note in passing that such an approach relegates Lagrange inversion of a power series to a special case of Möbius inversion.

As an application of these ideas to classical enumerating functions, we then consider the chromatic polynomial of a graph $G$. This we generalise to the bond polynomial, in which the substitution of an umbral integer $m \varphi$ yields information not only on the number of colourings of $G$ by $m$ colours, but also on their partition type. The new polynomial is visibly more powerful than the old, as we show by simple examples. In fact, in collaboration with $\mathrm{C}$. Wright [17], we have recently obtained a much superior generalisation along similar lines.

At this point we should note that the statement and interpretation of such results requires a working familiarity with the procedures of the original umbral calculus, as pioneered by $\mathrm{J}$. Blissard [3]. Indeed, we use his notation, as adapted by $\mathrm{J}$. Riordan [18], throughout. Thus has the wheel turned full circle!

We have arranged our sections so as to review $\Delta$-operators and binomial enumeration in $\S 1$ and $\S 2$ respectively. In $\S 3$ we develop the basic ideas of partition categories over $A$, which leads in $\S 4$ to a discussion of characteristic and conjugate polynomials, by way of Möbius functions.

In $\S 5$ we use this machinery to solve the realisation problem for all binomial sequences, and in $\S 6$ we introduce the bond polynomial of a graph.

It is a pleasure to acknowledge the encouragement given by $\mathrm{N}$. Biggs, E. K. Lloyd, G.-C. Rota and C. Wright during the preparation of this work.

1. $\Delta$-operators over a ring. We begin by reviewing some basic notation, and extending the fundamentals of the language and theory of $\Delta$-operators. General references are $[\mathbf{1}, \mathbf{1 5}$ and 19].

Let $A$ be a commutative ring with 1 , free of additive torsion. Often, we shall require $A$ to be graded by an integer valued function, usefully construed as complex dimension, and write $A$ as $A_{*}$ when we wish to emphasise the grading.

Let $A[x]$ denote the binomial coalgebra over $A$ in the 1-dimensional indeterminate $x$. This is free over $A$ on generators $\left\{1, x, x^{2}, \ldots\right\}$, and has coproduct

$$
x^{n} \mapsto \sum_{j=0}^{n}\left(\begin{array}{l}
n \\
j
\end{array}\right) x^{n-j} \otimes x^{j} .
$$

Then $D=d / d x$ is a linear operator on $A[x]$, and may itself be assigned dimension 1. This permits us to identify the continuous dual of $A[x]$ with the ring $A((D))$ of formal divided power series under multiplication, free over $A$ on $\left\{1, D, D^{2} / 2 !, \ldots\right\}$, and known as the umbral algebra. The duality is expressed by

$$
\left\langle D^{m} / m ! \mid x^{n}\right\rangle=\left\langle 1 \mid\left(D^{m} / m !\right) x^{n}\right\rangle=\left.\left(D^{m} / m !\right) x^{n}\right|_{x=0}=\delta_{m, n} .
$$

In more traditional examples of these concepts, $A$ is usually one of the fields $\mathbf{Q}$, $\mathbf{R}$ or $\mathbf{C}$.

We now select an umbra, or sequence $\alpha=\left(1, \alpha_{1}, \alpha_{2}, \ldots\right), \alpha_{i} \in A_{i}$, and write

$$
e^{\alpha D} \in A((D)), \quad \alpha^{i} \equiv \alpha_{i-1},
$$

for the 1-dimensional operator

$$
1+D+\alpha_{1} D^{2} / 2 !+\cdots+\alpha_{i-1} D^{i} / i !+\cdots
$$


This is Blissard's original "representative notation" [3]. The formula

$$
e^{\alpha D} x^{n}=(x+\alpha)^{n}, \quad \alpha^{i} \equiv \alpha_{i-1},
$$

holds, and may be linearly extended to all $p(x) \in A[x]$. Hence

$$
\left\langle e^{\alpha D} \mid p(x)\right\rangle=\left.p(x+\alpha)\right|_{x=0}=p(\alpha), \quad \alpha^{i} \equiv \alpha_{i-1},
$$

which we interpret as the umbral substitution of $x$ by $\alpha$.

(1.2) Definition. We call an operator of the form $e^{\alpha D}-1$ a $\Delta$-operator over $A$, and may alternatively write it as $\Delta$, or $\alpha(D)$.

Since

$$
\Delta p(x)=p(x+\alpha)-p(x), \quad \alpha^{i} \equiv \alpha_{i-1},
$$

$\Delta$ represents the umbral difference operator. Such $\Delta$-operators are the essence of the Roman-Rota umbral calculus.

(1.3) PROPOSITION. The set of all $\Delta$-operators over $A$ forms a group $\Delta(A)$ under composition, with identity $D$.

This is standard algebra, and the only subtlety involved is in the existence of an inverse, or conjugate $\Delta$-operator

$$
\bar{\Delta}=\bar{\alpha}(D)=e^{\bar{\alpha} D}-1
$$

satisfying $\bar{\alpha}(\alpha(D))=\alpha(\bar{\alpha}(D))=D$. The umbra $\bar{\alpha}$ is given explicitly by the Lagrange inversion principle $[\mathbf{2 2}, \mathbf{4}]$

$$
\bar{\alpha}_{i-1}=\left\langle(\Delta / D)^{-i} x^{i-1}\right\rangle \text { in } A .
$$

To each $\Delta$-operator, we assign the sequence

$$
a_{*}(x)=\left(a_{0}(x), a_{1}(x), \ldots\right)
$$

of (unnormalised) associated polynomials, where each $a_{n}(x) \in A_{n}[x]$ is monic of degree $n$. These are characterised by the properties

$$
\begin{gathered}
a_{0}(x)=1, \quad a_{n}(0)=0, \quad n>0 \\
\Delta a_{n}(x)=n a_{n-1}(x), \quad n>0,
\end{gathered}
$$

and always constitute a binomial sequence since, in $A[x, y]$,

$$
a_{n}(x+y)=\sum_{j=0}^{n}\left(\begin{array}{l}
n \\
j
\end{array}\right) a_{n-j}(x) a_{j}(y), \quad n>0 .
$$

This extends to the multinomial property

$$
a_{n}\left(x_{1}+\cdots+x_{s}\right)=\sum\left(\begin{array}{c}
n \\
k_{1}, \ldots, k_{s}
\end{array}\right) a_{k_{1}}\left(x_{1}\right) \cdots a_{k_{s}}\left(x_{s}\right)
$$

by induction.

Two monic binomial sequences $a_{*}(x), b_{*}(x)$ over $A$ may be combined to give a third by the umbral composition

$$
a b_{n}(x)=a_{n}\left(b_{*}(x)\right), \quad\left(b_{*}(x)\right)^{i} \equiv b_{i}(x),
$$

for all $n$. 
(1.5) Proposition. The set of all monic binomial sequences over $A$ forms a group $\mathrm{bi}(A)$ under umbral composition, with identity $x_{*}=\left(1, x, x^{2}, \ldots\right)$.

Once more this is standard algebra, best done by induction; we write the inverse of $a_{*}(x)$ as $\bar{a}_{*}(x)$ and refer to it as the (unnormalised) conjugate sequence of $\Delta$.

One of the main contributions of Mullin and Rota may be expressed as

(1.6) ThEOREM. The map $s: \Delta(A) \rightarrow \operatorname{bi}(A)$ specified by $\alpha(D) \mapsto a_{*}(x)$ is an isomorphism of groups.

The proof includes the observation that, if $\alpha(D), \beta(D)$ have respective associated sequences $a_{*}(x), b_{*}(x)$, then $\alpha(\beta(D))$ has associated sequence $a b_{*}(x)$. One of its consequences is that $\bar{a}_{*}(x)$ is the associated sequence of $\bar{\Delta}$.

The isomorphism $s$ involves the fundamental fact of umbral calculus:

$$
e^{a_{*}(x) D}=e^{x \bar{\Delta}}, \quad\left(a_{*}(x)\right)^{i} \equiv a_{i}(x),
$$

in $A[x]((D))$. Thus, given a monic binomial sequence

$$
a_{n}(x)=\sum_{k=1}^{n} a_{n, k} x^{k}
$$

if we define its umbra $\alpha$ by $\alpha=\left(1, a_{2,1}, a_{3,1}, \ldots\right)$ then it is the conjugate sequence for $\alpha(D)$. This suggests it is more natural to consider

$$
\bar{s}: \Delta(A) \rightarrow \operatorname{bi}(A),
$$

specified by $\alpha(D) \mapsto \bar{a}_{*}(x)$, an isomorphism by (1.6).

As a final comment, we note that an alternative version of (1.6) can be given by referring to [2 and $\mathbf{8}]$.

We let a binomial matrix over $A$ be a doubly infinite matrix $a=\left(a_{n, k}\right)$, with $a_{n, k} \in A_{n-k}$, which satisfies

(i) $a$ is lower triangular, with $a_{n, n}=1$,

(ii) $a d=d a^{\otimes 2}$

where $a^{\otimes 2}$ is the tensor square of $a$, indexed by $a_{(i, j),(l, m)}^{\otimes 2}$, and $d$ is the coproduct matrix given by

$$
d_{k,(i, j)}= \begin{cases}\left(\begin{array}{l}
i \\
j
\end{array}\right) & \text { if } i=k-j, \\
0 & \text { otherwise. }\end{cases}
$$

(1.10) PROPOSITION. The set of all binomial matrices over $A$ forms a group $m x(A)$ under matrix multiplication, with identity the infinite identity matrix.

The proof is again standard algebra. We write the inverse of $a$ as $\bar{a}$ and also refer to it as the conjugate of $a$.

(1.11) THEOREM. The map $m: \operatorname{bi}(A) \rightarrow m x(A)$ specified by $a_{*}(x) \mapsto a$ is an isomorphism of groups.

The proof includes the remark that the matrix of an umbral composition is the product of the corresponding matrices. It then follows that $\bar{a}_{*}(x)$ has matrix $\bar{a}$, hence the notation and nomenclature.

Combining (1.6) and (1.11), we can label $a$ and $\bar{a}$ as the associated and conjugate matrices of $\Delta$ respectively.

We give several examples of the above concepts in the next section. 
2. Binomial enumeration. We now give a swift survey of our version of the theory of binomial enumeration, whose foundations have been laid in $[9,13]$.

Recall from [9] that a species $B$ is a covariant endofunctor on the category of finite sets and bijections. For each finite set $E$, an element $s \in B(E)$ is a $B$-structure on $E$. We give two examples, to which we shall return, as illustration.

(2.1) EXAMPLES. (i) Let $T$ be the species of rooted trees, so that $t \in T(E)$ is a rooted tree with vertex set $E$.

(ii) Let $C$ be the species of complete graphs, so that $c \in C(E)$ is a complete graph with vertex set $E$.

Following Nava and Rota [14], let $\Pi(E)$ be the poset of all partitions $\pi$ of $E$, ordered by refinement, and write $|\pi|$ for the number of blocks of $\pi$.

(2.2) Definition. A k-assembly of $B$-structures on $E$ is a partition $\pi \in \Pi(E)$, with $|\pi|=k$ and such that each block of $\pi$ is endowed with a $B$-structure. We let $B_{k}(E)$ denote the set of all such $k$-assemblies.

We note there is a bijection between $B_{k}(E)$ and $B_{k}(\mathbf{n})$, where $|E|=n$ and $\mathbf{n}=\{1,2, \ldots, n\}$.

Returning to (2.1), a $k$-assembly of $T$-structures on $E$ is a $k$-forest of rooted trees with vertex set $E$, whilst a $k$-assembly of $C$-structures on $E$ is a disjoint union of complete graphs with vertex set $E$. Henceforth, as in these examples, we assume that

$$
\begin{aligned}
& B(\varnothing)=\{\varnothing\} \text { and }|B(\mathbf{1})|=1 \\
& B_{0}(E)=\varnothing \text { if } E \neq \varnothing .
\end{aligned}
$$

We are interested in enumerating assemblies of $B$-structures, so we define sequences of nonnegative integers by

(2.4) Definition.

$$
b_{n, k}= \begin{cases}\left|B_{k}(\mathbf{n})\right|, & k \leq n, \\ 0, & k>n .\end{cases}
$$

By $(2.3), b_{0,0}=1 ; b_{n, 0}=0, n>0 ; b_{n, n}=1$.

Note that $b_{n, 1}$ is simply the number of $B$-structures on $\mathbf{n}$, and it is convenient to introduce the umbra

$$
\beta=\left(1, \beta_{1}, \beta_{2}, \ldots\right), \quad \beta_{n-1}=b_{n, 1} .
$$

(2.6) THEOREM (MULLIN AND ROTA). If $b_{n}(x)=\sum b_{n, k} x^{k}$ in $\mathbf{Z}[x]$ is the enumerator for assemblies of $B$ structures on $\mathbf{n}$, then $b_{*}(x)$ is binomial over $\mathbf{Z}$.

Note that (1.6) now implies that $b_{*}(x)$ is the conjugate sequence for $\Delta=e^{\beta D}-1$.

We provide our own proof of $(2.6)$ in $\S 5$, where it will appear as a special case of a more general result.

Returning to our guiding examples (2.1), we may apply Cayley's theorem that $t_{n, 1}=n^{n-1}$ to (2.6), and deduce that the enumerators $t_{n}(x)$ for forests of rooted trees on $n$ vertices make up the conjugate sequence for $\Delta=\sum n^{n-1} D^{n} / n$ !. Hence, for example by consulting [19], we deduce that $t_{n}(x)$ is the $n$th Abel polynomial, i.e.

$$
t_{n}(x)=x(x+n)^{n-1} \text {. }
$$

In fact in [13], Mullin and Rota reverse this argument. They employ simple combinatorial properties of forests of rooted trees to show that $t_{*}(x)$ is the associated 
sequence for $\Delta=D e^{-D}$. This implies that the Abel polynomials are the soughtafter enumerators, and hence provides simultaneous and elegant proof that they are binomial, and that Cayley's theorem holds. Such arguments reveal the versatility of this approach.

In the case $B=C, c_{n, 1}$ is clearly 1 , so that the enumerators $c_{n}(x)$ for unions of complete graphs, or partitions of $\mathbf{n}$, make up the conjugate sequence for $\Delta=$ $\log (1+D)$. Thus, consulting [19] again, $c_{n}(x)$ is the $n$th exponential polynomial

$$
c_{n}(x)=\sum_{k} S(n, k) x^{k},
$$

where the $S(n, k)$ are the Stirling numbers of the second kind.

The idea behind Theorem (2.6) is to try and realise a binomial sequence $b_{*}(x)$ in some concrete fashion. Thus, if we think of $x$ as an integral variable, say as the cardinality of a finite set $X$, we may interpret $b_{n}(x)$ as the number of assemblies of $B$-structures on $\mathbf{n}$, where each block carries a label from $X$. From this viewpoint, the binomiality of $b_{*}(x)$ is straightforward.

However, such a description is even in principle only available if $b$ is a matrix of nonnegative integers, and so excludes, for example, the case of the falling factorial polynomials

$$
[x]_{n}=x(x-1) \cdots(x-n+1)=\sum_{k} s(n, k) x^{k},
$$

where the $s(n, k)$ are the Stirling numbers of the first kind. Mullin and Rota hint at a generalisation of their theory to incorporate such cases, and it is one of our purposes here to implement such a programme.

3. Partition categories. In this section, we develop the language of partitions, partition types and partition categories. Our methods are suggested by those of Content, Lemay and Leroux [5], but differ from theirs in detail.

We keep two main aims in mind.

The first is to state and prove our generalisation of Theorem (2.6) by giving a concrete realisation of all conjugate and associated sequences. The second is to define and study type algebras in $\S 4$.

We begin by recalling the poset $\Pi(E)$ of $\S 2$, and some related terminology from [14]. Sinch each $\pi \in \Pi(E)$ is itself a set of blocks, whenever $\pi \leq \sigma$ we can define the induced partition $\sigma / \pi \in \Pi(\pi)$. Similarly, to each $\tau \in \Pi(\pi)$ we may associate the coinduced partition $\tau \cdot \pi \in \Pi(E)$ by remembering that the blocks of $\pi$ are made up of elements of $E$. So

$$
(\sigma / \pi) \cdot \pi=\sigma \text { in } \Pi(E), \quad(\tau \cdot \pi) / \pi=\tau \text { in } \Pi(\pi) .
$$

We now turn $\pi(E)$ into a category, considered as a collection of arrows with partial composition law as in Mac Lane [12]. Thus to each refinement $\pi \leq \sigma$ in $\Pi(E)$ we assign an indecomposable arrow $(\pi, \sigma)$, and allow these to freely generate arrows of the form $(\pi, \sigma)(\sigma, \tau) \cdots(\nu, \zeta)$, composed by juxtaposition where permissible. We label the resulting category $P(E)$; it is a Möbius category [5].

Observe that $P(E)$ has as objects the identity arrows $(\pi, \pi)$, written simply as $\pi$. Thus it has an initial object 0 consisting of the partition of $E$ into singletons, and final object 1 consisting of the single block $E$. 
For each arrow $(\pi, \sigma) \in \mathcal{P}(E)$, we can define the class of $(\pi, \sigma)$ to be the sequence $\lambda=\left(\lambda_{1}, \lambda_{2}, \ldots\right)$ of nonnegative integers such that $\lambda_{i}$ is the number of blocks of size $i$ in the partition $\sigma / \pi$, for $1 \leq i \leq|\pi|$. Thus

$$
\sum_{i \geq 1} i \lambda_{i}=|\pi|
$$

and

$$
\sum_{i \geq 1} \lambda_{i}=|\sigma|
$$

Now let $\varphi_{1}, \varphi_{2}, \ldots$ be independent variables, generating the free commutative monoid $F^{+} \varphi$. This may be taken as the category with a single object, and an arrow for each word in the generators.

(3.1) Definition. We call the word $\varphi_{1}^{\lambda_{2}} \varphi_{2}^{\lambda_{3}} \cdots \varphi_{|\pi|-1}^{\lambda_{|\pi|}} \in F^{+} \varphi$ the type of $(\pi, \sigma)$. In particular, if $\pi=0$, we abbreviate the type of $(0, \sigma)$ to the type of $\sigma$. If a $k$ assembly of $B$-structures on $E$ has underlying partition $\pi$, we refer to the type of $\pi$ as the type of the assembly.

Note that $(\pi, \sigma) \in P(E)$ has the same type as $\sigma / \pi$ in $P(\pi)$.

Our definition of the type differs from others in the literature $[\mathbf{1}, \mathbf{1 4}]$ in that it does not explicitly record the number of blocks which remain unamalgamated; we have set $\varphi_{0}=1$. This fact simplifies our applications.

We extend the notion of the type to every element of $P(E)$ by multiplicativity, and so obtain a functor $t: P(E) \rightarrow F^{+} \varphi$. Whenever necessary, we grade $F^{+} \varphi$ by assigning dimension $n$ to $\varphi_{n}$, so that $t(\pi, \sigma)$ has dimension $|\pi|-|\sigma|$.

Now let $A$ be any commutative ring, possibly graded and with identity.

(3.2) DEFINITION. We say that the category $\mathcal{P}(E)$ lies over $A$ if there is given a multiplicative identity preserving weight function $\omega$, which admits a factorisation

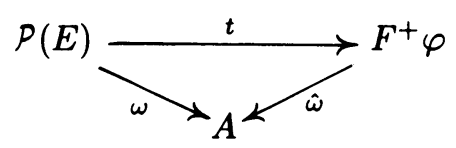

Here $\hat{\omega}$ is also assumed to be multiplicative, and we call it the classifying map for $\mathcal{P}(E)$ over $A$. If $A$ is graded, we require both $\omega$ and $\hat{\omega}$ to preserve dimension.

In general, $\hat{\omega}$ is not unique unless we insist that it be zero on each $\varphi_{n}$ not in the image of $t$. On the other hand, once a classifying map is chosen, the weight function is uniquely determined.

If $\mathcal{P}(E)$ and $\mathcal{P}\left(E^{\prime}\right)$ both lie over $A$, and have the same classifying map, we say they are compatible.

(3.3) EXAMPLES. (i) Suppose $A$ is the graded polynomial ring

$$
\Phi_{*}=\mathbf{Z}\left[\varphi_{1}, \varphi_{2}, \ldots\right],
$$

which we refer to as the ring of formal types. The weight function $\varsigma^{\varphi}: \mathcal{P}(E) \rightarrow \Phi_{*}$ is given by the same formula as $t$, and we call it the zeta type-function for $P(E)$, for reasons which will emerge in $\S 4$. The classifying map $\hat{\zeta}^{\varphi}$ simply includes $F^{+} \varphi$ as the monomials of $\Phi_{*}$. This is the generic, or universal example for $P(E)$, and unless otherwise stated, we shall assume that $P(E)$ lies over $\Phi_{*}$ as described. 
(ii) Suppose $A=\mathbf{Z}$, and the classifying map $\hat{\gamma}$ is specified by $\varphi_{i} \mapsto 1, i=$ $0,1,2, \ldots$. We call the resulting weight function $\gamma: \mathcal{P}(E) \rightarrow \mathbf{Z}$ the genus of $\mathcal{P}(E)$; it will feature extensively in later sections.

Whenever $\mathcal{P}(E)$ lies over $A$, we have the extended classifying map $\hat{\omega}: \Phi_{*} \rightarrow A$, which makes the following diagram commute:

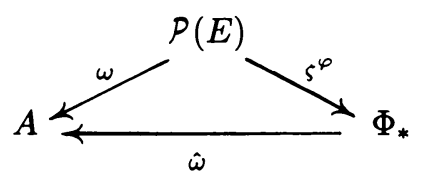

We can now formulate our fundamental concept.

(3.5) Definition. A partition category over $A$ is a subcategory $P$ of some $\mathcal{P}(E)$ which lies over $A$.

Thus every indecomposable arrow $f \in P$ has a representation $(\pi, \sigma)$ for $\pi \leq \sigma$ in some $\Pi(E)$, with composition given accordingly. Further, $f$ has a corresponding type in $F^{+} \varphi$, and weight $\omega(f)$ in $A$.

A morphism between two compatible partition categories over $A$ is a functor $P: P_{1} \rightarrow P_{2}$ which preserves types, and therefore weights, of arrows. An isomorphism is a morphism with an inverse.

When displaying partition categories it is useful to omit singletons from the notation for each $\pi$, so $0 \in \mathcal{P}(E)$ becomes $\varnothing$, and to label each indecomposable arrow with its weight.

(3.6) EXAMPLE. The chain category

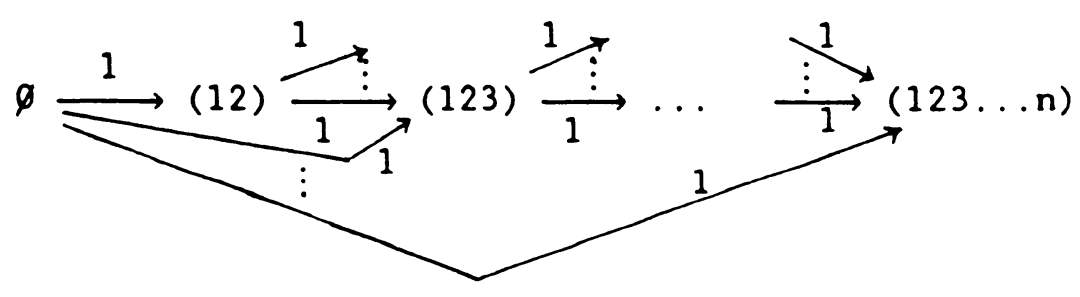

over any ring $A$.

Many more examples will be studied in detail in $\S \S 5$ and 6 .

One particular source of these arises by starting with $(\pi, \tau) \in P(E)$ and considering the full subcategory $P(\pi, \tau)$ of $P(E)$ generated by all arrows $(\rho, \sigma)$, where $\pi \leq \rho \leq \sigma \leq \tau$. We shall end this section by determining the structure of such examples.

(3.7) LEMMA. If $P(E)$ and $P\left(E^{\prime}\right)$ are compatible over $A$, then their product is a partition category over $A$ with the product weight function $\omega\left(\left(f, f^{\prime}\right)\right)=\omega(f) \omega\left(f^{\prime}\right)$.

PrOOF. Disjoint union defines an inclusion functor

$$
\amalg: P(E) \times P\left(E^{\prime}\right) \rightarrow P\left(E \amalg E^{\prime}\right)
$$

by

$$
\left((\pi, \sigma),\left(\pi^{\prime}, \sigma^{\prime}\right)\right) \mapsto\left(\pi \amalg \pi^{\prime}, \sigma \amalg \sigma^{\prime}\right) .
$$


This may be incorporated into the diagram

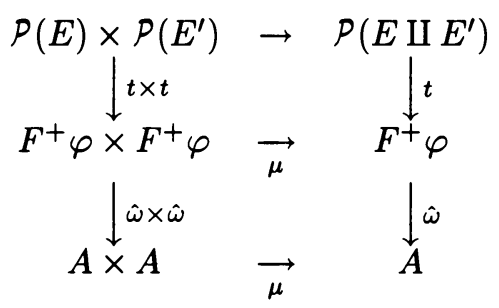

where each $\mu$ is multiplication.

The top square commutes by the definition of type, and the bottom because $\hat{\omega}$ is multiplicative. The result follows. $\Phi_{*}$.

(3.8) LEMMA. The partition categories $P(\pi)$ and $P(\pi, 1)$ are isomorphic over

Proof. Define a functor $F: P(\pi) \rightarrow P(\pi, 1)$ by $F((\rho, \sigma))=(\rho \cdot \pi, \sigma \cdot \pi)$. Then the diagram

commutes by definition of $t$.

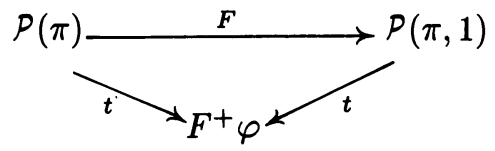

Thus $P(\pi)$ and $P(\pi, 1)$ are compatible over $\Phi_{*}$, and $F$ admits an inverse given by $F^{-1}((\rho, \sigma))=(\rho / \pi, \sigma / \pi)$.

Finally, let $\tau=\left\{E_{1}, \ldots, E_{|\tau|}\right\}$ in $\mathcal{P}(E)$, and for each $\pi \leq \tau$ write $\pi_{i} \in \mathcal{P}\left(E_{i}\right)$, $i=1, \ldots,|\tau|$, for the partition given by restricting $\pi$ to $E_{i}$.

(3.9) PROPOSITION. There is an isomorphism $P\left(\pi_{1}\right) \times \cdots \times P\left(\pi_{|\tau|}\right) \rightarrow P(\pi, \tau)$ of partition categories over $\Phi_{*}$.

PROOF. There is an isomorphism

$$
P\left(\pi_{1}\right) \times \cdots \times P\left(\pi_{|\tau|}\right) \stackrel{\times F}{\rightarrow} P\left(\pi_{1}, 1\right) \times \cdots \times P\left(\pi_{|\tau|}, 1\right)
$$

over $\Phi_{*}$, by applying (3.7) and (3.8). Using (3.7) again gives a morphism $P\left(\pi_{1}, 1\right) \times$ $\cdots \times P\left(\pi_{|\tau|}, 1\right) \rightarrow P(\pi, \tau)$ over $\Phi_{*}$ by $\left(\left(\rho_{1}, \sigma_{1}\right), \ldots,\left(\rho_{|\tau|}, \sigma_{|\tau|}\right)\right) \mapsto(\rho, \sigma)$ where $\rho=$ $\rho_{1} \amalg \cdots \amalg \rho_{|\tau|}$ and $\sigma=\sigma_{1} \amalg \cdots \amalg \sigma_{|\tau|}$. This is both injective and surjective, and so combines with $\times F$ to give the required isomorphism.

(3.10) COROLLARY. If $P(\pi, \tau)$ is given over $A$, then the isomorphism of (3.9) is also over $A$, after investing the product with the product weight function, as in (3.7).

Implicit in the above proof is the fact that, for any $\sigma \leq \tau,(\sigma, \tau)$ has type

$$
\prod_{i=1}^{|\tau|} \varphi_{\left|\sigma_{i}\right|-1}
$$

Of course, (3.7) and (3.8) are partition categorical versions of a standard poset result, e.g. see $[1,2.67]$. 
4. Characteristic and conjugate polynomials. We are now able to begin our discussion of the incidence algebras associated to a given partition category $P$ over $A$. This leads on to the study of Möbius functions, and the characteristic and conjugate polynomials of $P$.

If we write $\Pi$ for the underlying poset of $P$, then for any commutative ring $R$ with identity, possibly graded, we may form an incidence algebra $R \Pi$ in the usual fashion (e.g. see [1]). We now interpret this algebra in terms of the category $P$, and label it $R(P)$ to emphasise this alternative description.

The elements of $R(P)$ are the multiplicative functions $\alpha: P \rightarrow R$, each of which is uniquely determined by its values on the indecomposable arrows $(\pi, \sigma) \in P$. We therefore specify the algebra operations by the rules

$$
\begin{gathered}
a \alpha(\pi, \sigma)=a(\alpha(\pi, \sigma)), \quad(\alpha+\beta)(\pi, \sigma)=\alpha(\pi, \sigma)+\beta(\pi, \sigma), \\
(\alpha * \beta)(\pi, \sigma)=\sum_{\pi \leq \tau \leq \sigma} \alpha(\pi, \tau) \beta(\tau, \sigma)
\end{gathered}
$$

for any $\alpha, \beta \in R(P)$ and $a \in R$. The product $*$ is known as convolution, and the identity $\delta$ in $R(P)$ is given by $\delta \pi=1, \delta(\pi, \sigma)=0$ for $\pi \neq \sigma$.

We shall think of $A(P)$ as the incidence algebra of $P$; it contains the weight function $\omega$ as a distinguished element. When $R=\Phi_{*}$, the ring of formal types, we call $\Phi_{*}(P)$ the type algebra of $P$. The zeta type-function $\zeta^{\Phi}$ of $(3.3)$ naturally lies in $\Phi_{*}(P)$.

Observe that the extended classifying map $\hat{\omega}: \Phi_{*} \rightarrow A$ induces a homomorphism $\hat{\omega}_{*}: \Phi_{*}(P) \rightarrow A(P)$ which satisfies $\hat{\omega}_{*}\left(\varsigma^{\varphi}\right)=\omega$. In particular, if $\omega$ is the genus $\gamma: P \rightarrow \mathbf{Z}$ of $(3.3(\mathrm{ii}))$, then $\hat{\gamma}_{*}\left(\varsigma^{\varphi}\right)$ is the classical zeta function of $\Pi$ (e.g. see [1] ).

(4.1) LEMMA. An element $\alpha \in R(P)$ is invertible under convolution iff $\alpha \pi$ is invertible in $R$ for each identity $\pi \in P$.

PROOF. The formula for such an $\alpha^{-1}$ has to be given inductively by

$$
\alpha^{-1} \pi=1 / \alpha \pi, \quad \alpha^{-1}(\pi, \sigma)=-\sum_{\pi \leq \tau<\sigma} \alpha^{-1}(\pi, \tau) \alpha(\tau, \sigma) / \alpha \sigma .
$$

(4.2) COROLLARY. The zeta type-function $\varsigma^{\varphi} \in \Phi_{*}(P)$ and the weight function $\omega \in A(P)$ are both invertible, since they assign the value 1 to every identity.

We shall be much concerned with the inverse of these two functions, which we now characterise in an alternative and combinatorially attractive fashion. For this purpose, we describe functions $\zeta_{-}^{\varphi} \in \Phi_{*}(P)$ and $\omega_{-} \in A(P)$ as follows;

$$
\zeta_{-}^{\varphi}(\pi, \sigma)=-\zeta^{\varphi}(\pi, \sigma), \quad \omega_{-}(\pi, \sigma)=-\omega(\pi, \sigma)
$$

for each indecomposable arrow $(\pi, \sigma) \in P$, whilst $\zeta_{-}^{\varphi} \pi=1$ and $\omega_{-} \pi=1$.

(4.3) Definition. The Möbius type-function $\mu^{\varphi} \in \Phi_{*}(P)$ is defined by

$$
\mu^{\varphi}(\pi, \sigma)=\sum_{f: \pi \rightarrow \sigma} \zeta_{-}^{\varphi} f,
$$

and the Möbius weight function $\mu \in A(P)$ by

$$
\mu(\pi, \sigma)=\sum_{f: \pi \rightarrow \sigma} \omega_{-} f
$$


Of course, all the above functions are by definition extended to arbitrary arrows using multiplicativity.

As a consequence of Definition (4.3), and in tandem with (3.4), there is a commutative triangle

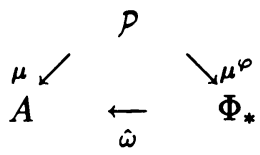

Thus $\hat{\omega}_{*}\left(\mu^{\varphi}\right)=\mu$. In particular, $\hat{\gamma}_{*}\left(\mu^{\varphi}\right)$ is the classical Möbius function of $\Pi$ (e.g. see $[\mathbf{1}])$.

(4.4) PROPOSITION. The zeta type-function $\varsigma^{\varphi}$ and the weight function $\omega$ have convolution inverse $\mu^{\varphi}$ and $\mu$ respectively.

ProOF. Each $f: \pi \rightarrow \sigma$ can be expressed uniquely as a composition $f=g h$, where $h: \pi \rightarrow \tau$ and $g=(\tau, \sigma)$ for some $\tau<\sigma$. Thus

$$
\begin{aligned}
\mu^{\varphi}(\pi, \sigma) & =-\sum_{\pi \leq \tau<\sigma}\left(\sum_{h: \pi \rightarrow \tau} \zeta_{-}^{\varphi} h\right) \varsigma^{\varphi}(\tau, \sigma) \\
& =-\sum_{\pi \leq \tau<\sigma} \mu^{\varphi}(\pi, \tau) \varsigma^{\varphi}(\tau, \sigma) .
\end{aligned}
$$

But by Corollary (4.2), this is precisely the formula for $\left(\varsigma^{\varphi}\right)^{-1}$.

Similarly,

$$
\mu(\pi, \sigma)=-\sum_{\pi \leq \tau<\sigma} \mu(\pi, \tau) \omega(\tau, \sigma)
$$

so $\mu=\omega^{-1}$.

We now describe a faithful matrix representation of $R(P)$. This requires us to choose an indexing $\left\{\pi_{i}: i=1,2, \ldots, m\right\}$ of the identities of $P$, with the property that $\left(\pi_{i}, \pi_{j}\right) \in P \Leftrightarrow i \geq j$. We may do this by selecting a total extension of the underlying dual partial ordering of $\Pi$.

Now let $\operatorname{LT}(m, R)$ be the algebra of $m \times m$ lower triangular matrices with entries in $R$, and assign to every $\alpha \in R(P)$ the matrix

$$
M(\alpha)_{i, j}= \begin{cases}\alpha\left(\pi_{i}, \pi_{j}\right), & i \geq j, \\ 0, & \text { otherwise. }\end{cases}
$$

(4.5) PROPOSITION. The map $M: R(P) \rightarrow \mathrm{LT}(m, R)$ is a faithful algebra representation.

PrOOF. Clearly $M$ is a monomorphism of $R$-modules, and preserves identities. It is multiplicative, since

$$
M(\alpha * \beta)_{i, j}=\sum_{k} \alpha\left(\pi_{i}, \pi_{k}\right) \beta\left(\pi_{k}, \pi_{j}\right)=M(\alpha) M(\beta) .
$$

Since the matrices $M\left(\varsigma^{\varphi}\right)$ and $M(\omega)$ are so useful, we call them the zeta typematrix and the weight matrix of $P$, and denote them by $Z^{\varphi}$ and $W$ respectively. Similarly, we call $M\left(\mu^{\varphi}\right)$ and $M(\mu)$ the Möbius type-matrix and the Möbius matrix 
of $P$, and label them $M^{\varphi}$ and $M$ respectively. Hence, by Proposition (4.4) and (4.5),

$$
\begin{array}{ll}
M^{\varphi}=\left(Z^{\varphi}\right)^{-1} & \text { in } \operatorname{LT}\left(m, \Phi_{*}\right), \\
M=W^{-1} & \text { in } \operatorname{LT}(m, A) .
\end{array}
$$

Thus if $\omega$ is the genus $\gamma$, the matrices $W$ and $M$ are the usual zeta and Möbius matrices of $\Pi$ (e.g. see [11]).

Let us now take $\mathbf{x}$ to be the column vector

$$
\mathbf{x}=\left[x^{\left|\pi_{1}\right|}, x^{\left|\pi_{2}\right|}, \ldots, x^{\left|\pi_{m}\right|}\right]^{T},
$$

where $x$ is an indeterminate, assigned dimension 1 in the graded situation. So $Z^{\varphi} \mathbf{X}$ and $M^{\varphi} \mathbf{X}$ are $m \times 1$ column vectors, whose respective $i$ th entries we write as $\bar{c}^{\varphi}\left(\pi_{i} ; x\right), c^{\varphi}\left(\pi_{i} ; x\right)$. These are polynomials of degree $\left|\pi_{i}\right|$ over $\Phi_{*}$. Similarly, the $i$ th entries of $W \mathbf{x}$ and $M \mathbf{x}$ are polynomials $\bar{c}\left(\pi_{i} ; x\right), c\left(\pi_{i} ; x\right)$ of degree $\left|\pi_{i}\right|$ over $A$.

(4.7) Definition. We call $\bar{c}^{\varphi}\left(\pi_{i} ; x\right)$ and $c^{\varphi}\left(\pi_{i} ; x\right)$ the conjugate and characteristic type-polynomials of $P$ at $\pi_{i}$ respectively. Similarly, we call $\bar{c}\left(\pi_{i} ; x\right)$ and $c\left(\pi_{i} ; x\right)$ the conjugate and characteristic polynomials of $P$ at $\pi_{i}$.

When $i=m$, so that $\pi_{m}$ is initial in $P$, we replace $\pi_{m}$ by $P$ in the notation, and simply refer, for example, to $c(P ; x)$ as the characteristic polynomial of $P$.

From the graded viewpoint, both $\bar{c}^{\varphi}\left(\pi_{i} ; x\right)$ and $c^{\varphi}\left(\pi_{i} ; x\right)$ lie in $\Phi_{\left|\pi_{i}\right|}[x]$, and if $A$ is graded, $\bar{c}\left(\pi_{i} ; x\right)$ and $c\left(\pi_{i} ; x\right)$ lie in $A_{\left|\pi_{i}\right|}[x]$.

Observe that the induced homomorphism $\hat{\omega}: \Phi_{*}[x] \rightarrow A[x]$ maps $\bar{c}^{\varphi}\left(\pi_{i} ; x\right)$ to $\bar{c}\left(\pi_{i} ; x\right)$, and $c^{\varphi}\left(\pi_{i} ; x\right)$ to $c\left(\pi_{i} ; x\right)$. In particular, when $\omega$ is the genus $\gamma$, then $c(P ; x)$ is the classical characteristic polynomial of the poset $\Pi$ (e.g. see [1]) multiplied by $x^{\left|\pi_{1}\right|}$.

The polynomials of Definition (4.7) have several properties of which we shall subsequently take advantage. As a first example, we explain how they act as enumerators for the category.

Consider the expansions

$$
\begin{gathered}
\bar{c}^{\varphi}\left(\pi_{i} ; x\right)=\sum_{k} \bar{c}_{i, k}^{\varphi} x^{k}, \quad c^{\varphi}\left(\pi_{i} ; x\right)=\sum_{k} c_{i, k}^{\varphi} x^{k}, \\
\bar{c}\left(\pi_{i} ; x\right)=\sum_{k} \bar{c}_{i, k} x^{k}, \quad c\left(\pi_{i} ; x\right)=\sum_{k} c_{i, k} x^{k},
\end{gathered}
$$

and let $R\left(\pi_{i}, k\right)$ denote the set of arrows $\left(\pi_{i}, \pi_{j}\right) \in \mathcal{P}$ with $\left|\pi_{j}\right|=k$.

(4.8) Proposition. The coefficient $\bar{c}_{i, k}^{\varphi} \in \Phi_{\left|\pi_{i}\right|-k}$ enumerates the elements of $R\left(\pi_{i}, k\right)$ by type.

PROOF. As an alternative definition, we have

$$
\bar{c}^{\varphi}\left(\pi_{i} ; x\right)=\sum_{\pi_{j} \geq \pi_{i}} \varsigma^{\varphi}\left(\pi_{i}, \pi_{j}\right) x^{\left|\pi_{j}\right|}
$$

thus

$$
\bar{c}_{i, k}^{\varphi}=\sum_{\substack{\pi_{j} \geq \pi_{i} \\\left|\pi_{j}\right|=k}} \varsigma^{\varphi}\left(\pi_{i}, \pi_{j}\right),
$$

as sought. 
Using the same mode of proof yields

(4.9) COROLlaRY. The coefficients $c_{i, k}^{\varphi}, \bar{c}_{i, k}, c_{i, k}$ enumerate the elements of $R\left(\pi_{i} ; k\right)$ by Möbius type, weight and Möbius weight respectively.

A second important property develops the concept of composition described in $\S 1$, and explains the sense in which conjugate and characteristic polynomials are mutually inverse. For each $\pi_{i}$ we define the umbral composition $\bar{c}^{\varphi} c^{\varphi}\left(\pi_{i} ; x\right)$ by

$$
\bar{c}^{\varphi}\left(\pi_{i} ; c^{\varphi}\left(\pi_{*} ; x\right)\right), \quad c^{\varphi}\left(\pi_{*} ; x\right)^{\left|\pi_{j}\right|} \equiv c^{\varphi}\left(\pi_{j} ; x\right) .
$$

(4.10) Proposition. $\bar{c}^{\varphi} c^{\varphi}\left(\pi_{i} ; x\right)=x^{\left|\pi_{i}\right|}$.

ProOF. By construction, $\bar{c}^{\varphi} c^{\varphi}\left(\pi_{i} ; x\right)$ is given by

$$
\sum_{i \geq j \geq k} \varsigma^{\varphi}\left(\pi_{i}, \pi_{j}\right) \mu^{\varphi}\left(\pi_{j}, \pi_{k}\right) x^{\left|\pi_{k}\right|},
$$

which is the $i$ th entry of $Z^{\varphi} M^{\varphi} x$. The result follows from (4.6).

The same mode of proof provides

(4.11) COROLLARY. For each $\pi_{i}$,

$$
c^{\varphi} \bar{c}^{\varphi}\left(\pi_{i} ; x\right)=x^{\left|\pi_{i}\right|}, \quad \bar{c} c\left(\pi_{i} ; x\right)=c \bar{c}\left(\pi_{i} ; x\right)=x^{\left|\pi_{i}\right|} .
$$

To conclude, we return to the notion of umbral substitution introduced in $\S 1$. For $t=0,1,2, \ldots$ we define the umbral integer $t$ by

$$
t \varphi=\overbrace{\varphi+\cdots+\varphi}^{t}
$$

so that for any $p(x) \in \Phi_{*}[x]$ we can evaluate $p(t \varphi) \in \Phi_{*}$ by repeated use of the multinomial theorem and $\varphi^{i} \equiv \varphi_{i-1}$. By (1.1), we have

$$
p(t \varphi)=\left\langle\left(e^{\varphi D}\right)^{t} \mid p(x)\right\rangle .
$$

We shall determine $(t \varphi)^{n}$ in $\S 5$.

Note that the classifying map $\hat{\omega}$ induces a homomorphism $\hat{\omega}: \Phi_{*}((D)) \rightarrow A((D))$ which maps $e^{\varphi D}$ to the umbral translation $e^{\alpha D}$ over $A$, with $\alpha_{i}=\hat{w}\left(\varphi_{i}\right)$. So by (4.12),

$$
\hat{\omega}(p(t \varphi))=(\hat{\omega} p)(t \alpha) .
$$

(4.14) Definition. We call the sequence of formal types $c^{\varphi}(P ; t \varphi), t=0,1$, $2, \ldots$, the characteristic types of $P$. We call the sequence of elements $c(P ; t \alpha) \in A$, $t=0,1,2, \ldots$, the characteristic weights of $P$.

These sequences will be important in $\S 6$, where we shall utilise the fact that, by (4.13), $\hat{\omega}$ maps the first sequence to the second. If $\omega$ is the genus $\gamma$, then the characteristic weights reduce to the integers $c(P ; t)$.

5. Realising binomial sequences. We now introduce our first application of partition categories, by extending the Mullin-Rota theory previewed in $\$ 2$.

(5.1) DEFINITION. A binomial enumeration problem over $A$ is a choice of weight function $\omega: \mathcal{P}(E) \rightarrow A$ for each finite set $E$, such that $\mathcal{P}(E)$ and $\mathcal{P}(F)$ are compatible over $A$ for each $E$ and $F$.

In order to give several crucial examples which relate this definition to $\$ 2$, we need an alternative characterisation. 
(5.2) LEMMA. A weight function $\omega$ determines a binomial enumeration problem iff

$$
\omega(\pi, \sigma)=\prod_{2 \leq i \leq|\sigma|} \omega_{i-1}^{\lambda_{i}}
$$

for all $(\pi, \sigma) \in \mathcal{P}(E)$, where $\omega_{i-1}=\omega(0,1)$ for $(0,1) \in \mathcal{P}(\mathbf{i})$, and $(\pi, \sigma)$ has class $\lambda$.

Proof. Suppose $\omega$ is a binomial enumeration problem. Since $P(E)$ has the same classifying map $\hat{\omega}: F^{+} \varphi \rightarrow A$ for all $E$, however large, it is completely determined by the values $\hat{\omega}\left(\varphi_{i-1}\right), i \geq 2$. These are in turn given by choosing $E=\mathbf{i}$, so that

$$
\hat{\omega}\left(\varphi_{i-1}\right)=\omega(0,1) \quad \text { for }(0,1) \in P(\mathbf{i}) .
$$

Now $(\pi, \sigma)$ has type $\prod_{2 \leq i \leq|\sigma|} \varphi_{i-1}^{\lambda_{i}}$, so by the multiplicativity of $\hat{\omega}, \omega(\pi, \sigma)$ has the stated form.

Conversely, if a sequence $\omega=\left(1, \omega_{1}, \omega_{2}, \ldots\right)$ is given in $A$, it extends to a multiplicative map $\hat{\omega}: F^{+} \varphi \rightarrow A$ by choosing $\hat{\omega}\left(\varphi_{n}\right)=\omega_{n}, n=1,2, \ldots$ This classifies $\mathcal{P}(E)$ over $A$, compatibly for all $E$, by using the given formula.

Remark that, using the notation of (3.11), the description of $\omega$ in (5.2) becomes

$$
\omega(\pi, \sigma)=\prod_{i=1}^{|\sigma|} \omega_{\left|\pi_{i}\right|-1} .
$$

(5.4) EXAMPLES. (i) Given a species $B$, we let $\omega(B): \mathcal{P}(E) \rightarrow \mathbf{Z}$ be the $B$ enumeration problem, defined by letting $\omega(B)(\pi, \sigma)$ be the number of $|\sigma|$-assemblies of $B$-structures on the blocks of $\pi$ whose underlying partition is $\sigma / \pi$. Since this obeys (5.3), with $\omega(B)_{n}$ being the number of $B$-structures on $\mathbf{n}+\mathbf{1}$, we have a binomial enumeration problem. 1.

Our guiding examples (2.1) are both of this type, with $\omega(T)_{n}=(n+1)^{n} ; \omega(C)_{n}=$

(ii) With the same data as (i), we let $\omega^{\varphi}(B): \mathcal{P}(E) \rightarrow \Phi_{*}$ be the B-typification problem, defined by letting $\omega^{\varphi}(B)(\pi, \sigma)$ be $\omega(B)(\pi, \sigma) \zeta^{\varphi}(\pi, \sigma)$. Since this obeys (5.3), with $\omega^{\varphi}(B)_{n}$ being $\omega(B)_{n} \varphi_{n}$, we have a binomial enumeration problem.

Such problems enumerate assemblies of $B$-structures by type, hence the name, and reduce to the corresponding enumeration problem under the genus $\gamma$. Our examples (2.1) yield

$$
\omega^{\varphi}(T)_{n}=(n+1)^{n} \varphi_{n} ; \quad \omega^{\varphi}(C)_{n}=\varphi_{n} .
$$

Thus $\omega^{\varphi}(C)$ is the zeta type-function, and hence is the generic, or universal binomial enumeration problem.

Binomial enumeration problems over the same ring may, in fact, be composed.

(5.6) DeFinition. Suppose given binomial enumeration problems $\nu$ and $\omega$ over $A$; then we call $\nu \circ \omega: \mathcal{P}(E) \rightarrow A$, defined by

$$
\nu \circ \omega(\pi, \sigma)=\sum_{\pi \leq \tau \leq \sigma} \nu(\pi, \tau) \omega(\tau, \sigma / \tau),
$$

the composition of $\nu$ and $\omega$. 

$A$.

(5.7) LEMMA. The composition $\nu \circ \omega$ is a binomial enumeration problem over

ProOF. We check that $\nu \circ \omega$ satisfies (5.3) by noting

$$
\begin{aligned}
\sum_{\pi \leq \tau \leq \sigma} \nu(\pi, \tau) \omega(\tau, \sigma / \tau) & =\sum_{\pi \leq \tau \leq \sigma} \nu\left(\pi_{1}, \tau_{1}\right) \cdots \nu\left(\pi_{|\sigma|}, \tau_{|\sigma|}\right) \omega\left(\tau_{1}, \sigma_{1} / \tau_{1}\right) \\
& \cdots \omega\left(\tau_{|\sigma|}, \sigma_{|\sigma|} / \tau_{|\sigma|}\right) \\
& =\prod_{1 \leq i \leq|\sigma|} \sum_{\pi_{i} \leq \tau_{i}} \nu\left(\pi_{i}, \tau_{i}\right) \omega\left(\tau_{i}, \sigma_{i} / \tau_{i}\right) \\
& =\prod_{1 \leq i \leq|\sigma|} \nu \circ \omega\left(\pi_{i}, \sigma_{i} / \pi_{i}\right) . \quad
\end{aligned}
$$

In the context of the examples of (5.4), given two species $B_{1}$ and $B_{2}$, then

$$
\omega\left(B_{1}\right) \circ \omega\left(B_{2}\right)=\omega\left(B_{1} B_{2}\right)
$$

where $B_{1} B_{2}$ is Joyal's composition of species. This follows since $\omega\left(B_{1} B_{2}\right)_{n-1}$ is by definition the number of $B_{1}$-structures on each assembly of $B_{2}$-structures on $\mathbf{n}$, and so is given by

$$
\sum_{\tau \in \mathcal{P}(\mathbf{n})} \omega\left(B_{1}\right)(0, \tau) \omega\left(B_{2}\right)(\tau, 1 / \tau)
$$

A similar argument establishes the corresponding formula for $\omega^{\varphi}$.

The composition (5.5) is visibly associative, and invests the set of all binomial enumeration problems over $A$, which we label $\operatorname{ep}(A)$, with a semigroup structure, whose identity is the trivial problem $\iota$, satisfying

$$
\iota(\pi, \sigma)= \begin{cases}0, & \pi \neq \sigma \\ 1, & \pi=\sigma .\end{cases}
$$

Our next concern is to show that $\operatorname{ep}(A)$ is, in fact, a familiar group. This involves the polynomials of $\S 4$.

(5.8) Definition. Given $\omega \in \operatorname{ep}(A)$, we call the sequences

$$
\left.\begin{array}{l}
\bar{c}_{n}(\omega ; x)=\bar{c}(\mathcal{P}(E) ; x) \\
c_{n}(\omega ; x)=c(\mathcal{P}(E) ; x)
\end{array}\right\}, \quad|E|=n=1,2, \ldots,
$$

the conjugate sequence and the characteristic sequence of $\omega$ respectively. By convention, we write $\bar{c}_{0}(\omega ; x)=c_{0}(\omega ; x)=1$.

These sequences are well defined by virtue of Lemma (5.2). Thus, for example,

$$
\bar{c}_{n}(\omega ; x)=\sum_{\pi \in \mathcal{P}(\mathbf{n})} \omega(0, \pi) x^{|\pi|}=\sum_{\pi} \omega_{1}^{\lambda_{2}} \cdots \omega_{n-1}^{\lambda_{n}} x^{|\pi|} .
$$

When $\omega$ is the universal binomial enumeration problem $\omega^{\varphi}(C)$ of $(5.5)$, then the conjugate and characteristic sequences are type-polynomials as in Definition (4.7).

We have also to remark that the umbral composition formulae of Proposition (4.10) reduce in this case to

$$
\begin{aligned}
& \bar{c}_{n}\left(\omega ; c_{*}(\omega, x)\right)=x^{n}, \quad c_{*}(\omega ; x)^{i} \equiv c_{i}(\omega ; x), \\
& c_{n}\left(\omega ; \bar{c}_{*}(\omega, x)\right)=x^{n}, \quad \bar{c}_{*}(\omega ; x)^{i} \equiv \bar{c}_{i}(\omega ; x),
\end{aligned}
$$

for $n=0,1,2, \ldots$. 
(5.11) Proposition. The sequence $\bar{c}_{*}(\omega ; x)$ is binomial over $A$.

ProOF. We have

$$
\begin{aligned}
\bar{c}_{n}(\omega ; x+y) & =\sum_{\pi \in \mathcal{P}(\mathbf{n})} \omega(0, \pi)(x+y)^{|\pi|} \\
& =\sum_{\pi} \sum_{\pi_{1} \amalg \pi_{2}=\pi} \omega\left(0, \pi_{1}\right) x^{\left|\pi_{1}\right|} \omega\left(0, \pi_{2}\right) x^{\left|\pi_{2}\right|} \\
& =\sum_{j=1}^{n}\left(\begin{array}{c}
n \\
j
\end{array}\right) \sum_{\pi_{1} \in \mathcal{P}(\mathbf{n}-\mathbf{J})} \omega\left(0, \pi_{1}\right) x^{\left|\pi_{1}\right|} \sum_{\pi_{2} \in \mathcal{P}(\mathbf{J})} \omega\left(0, \pi_{2}\right) x^{\left|\pi_{2}\right|} \\
& =\sum_{j=1}^{n}\left(\begin{array}{c}
n \\
j
\end{array}\right) \bar{c}_{n-j}(\omega ; x) \bar{c}_{j}(\omega ; x) .
\end{aligned}
$$

(5.12) Proposition. The map $\bar{c}: \operatorname{ep}(A) \rightarrow \operatorname{bi}(A)$ specified by $\bar{c}(\omega)=\bar{c}_{*}(\omega ; x)$ is an isomorphism of semigroups.

PROOF. Clearly $\bar{c}(\iota)=x_{*}$, so it remains to verify that $\bar{c}$ is a $1-1$ correspondence and a homomorphism.

By (5.2), $\omega$ determines and is determined by the sequence $\left(1, \omega_{1}, \omega_{2}, \ldots\right)$ which is the umbra of $\bar{c}_{*}(\omega ; x)$ by (5.9). Since the isomorphism (1.8) shows that every binomial sequence determines and is determined by its umbra, we deduce that $\bar{c}$ is indeed a bijection.

Now

$$
\begin{aligned}
\bar{c}_{n}(\nu \circ \omega ; x) & =\sum_{\pi} \nu \circ \omega(0, \pi) x^{|\pi|} \\
& =\sum_{\pi} \sum_{0 \leq \tau \leq \pi} \nu(0, \tau) \omega(\tau, \pi / \tau) x^{|\pi|}
\end{aligned}
$$

has umbra given by

$$
\bar{c}_{n, 1}(\nu \circ \omega ; x)=\sum_{\tau} \nu(0, \tau) \omega(\tau, 1 / \tau)
$$

as does

$$
\bar{c}_{n}\left(\nu ; \bar{c}_{*}(\omega ; x)\right)=\sum_{\pi \leq \tau} \nu(0, \tau) \omega(\tau, \pi / \tau) x^{|\pi / \tau|} .
$$

So $\bar{c}$ is indeed multiplicative.

(5.13) COROLlaRY. The semigroup $\operatorname{ep}(A)$ is a group, and each $\omega \in \operatorname{ep}(A)$ has a conjugate problem $\mu$ such that $\bar{c}_{*}(\mu ; x)=c_{*}(\omega ; x)$.

PROOF. The first part is immediate from (5.12), which furnishes each $\omega$ with an inverse $\mu$. By $(5.10), \bar{c}(\mu)=c_{*}(\omega ; x)$.

Of course, we have previously identified $\mu$ with the Möbius weight function associated to $\omega$, by virtue of (4.9). As in the case of (1.6) and (1.8), the map

$$
c: \operatorname{ep}(A) \rightarrow \operatorname{bi}(A)
$$

specified by $c(\omega)=c_{*}(\omega ; x)$ would be just as suitable an isomorphism in Proposition (5.12). Then $c(\mu)=\bar{c}_{*}(\omega ; x)$.

We can now prove our main result, which subsumes Theorem (2.6). 
(5.15) THEOREM. Any binomial sequence over $A$ can be realised as the enumerators of the weights of the objects of a binomial enumeration problem.

The conjugate sequence can then be realised as the enumerators of the corresponding Möbius weights.

Proof. Combine (4.8), (4.9), (5.12) and (5.13).

(5.16) EXAMPLES. (i) We have now fully confirmed our analysis in $\S 2$ of Examples (2.1), realising the Abel polynomials as the enumerators of forests of rooted trees, and the exponential polynomials as enumerators of partitions, or assemblies of complete graphs. In the language of (5.4)

$$
t_{*}(x)=\bar{c}_{*}(\omega(T) ; x) \quad \text { and } \quad c_{*}(x)=\bar{c}_{*}(\omega(C) ; x) .
$$

The conjugate Abel polynomials are given by (see [19])

$$
\bar{t}_{n}(x)=\sum_{k=1}^{n}\left(\begin{array}{l}
n \\
k
\end{array}\right)(-k)^{n-k} x^{k},
$$

whilst the conjugate exponential polynomials are the falling factorials $[x]_{*}$ of (2.7). This follows from (1.10) and the fact that the binomial matrices of Stirling numbers of the first and second kinds are conjugate (or see [19] again). So $\bar{t}_{*}(x)$ and $[x]_{*}$ respectively enumerate forests of rooted trees, and partitions, by Möbius weights; these can be computed from their numbers by the procedures of (4.3), and are easily illustrated. Thus

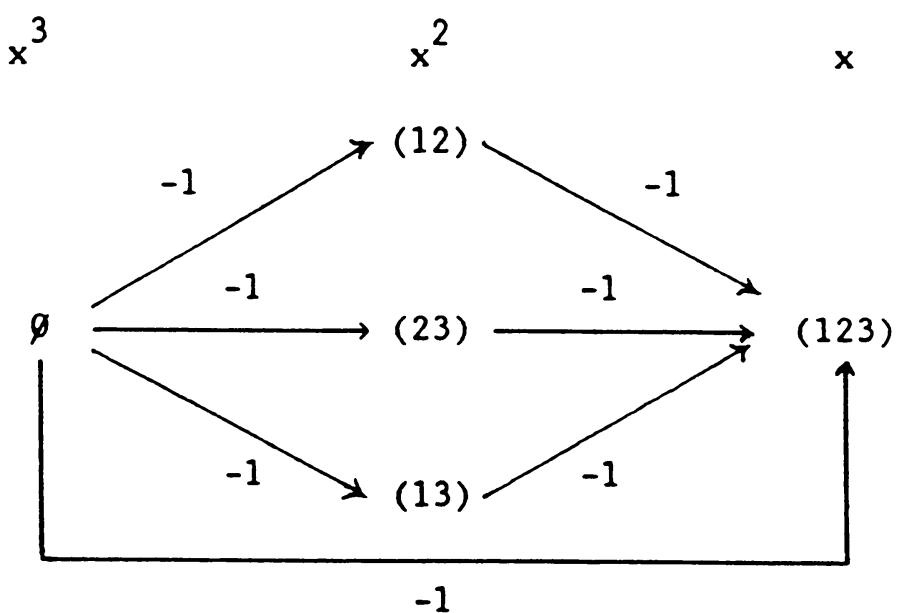

is a diagram for

$$
[x]_{3}=1 \cdot x^{3}+(-3) x^{2}+\left(3(-1)^{2}-1\right) x=x^{3}-3 x^{2}+2 x .
$$

(ii) The corresponding typification problems, as enunciated in (5.5), realise the Abel type-polynomials, expressible by (5.9) as

$$
t_{n}^{\varphi}(x)=\bar{c}_{n}\left(\omega^{\varphi}(T) ; x\right)=\sum_{\pi \in \mathcal{P}(\mathbf{n})} 2^{\lambda_{2}} 3^{2 \lambda_{3}} \cdots n^{(n-1) \lambda_{n}} \varphi_{1}^{\lambda_{2}} \cdots \varphi_{n-1}^{\lambda_{n}} x^{|\pi|}
$$


and their conjugates; and the exponential type-polynomials

$$
c_{n}^{\varphi}(x)=\bar{c}_{n}\left(\omega^{\varphi}(C) ; x\right)=\sum_{\pi \in \mathcal{P}(\mathbf{n})} \varphi_{1}^{\lambda_{2}} \cdots \varphi_{n-1}^{\lambda_{n}} x^{|\pi|}
$$

and their conjugates. The $c_{n}^{\varphi}(x)$ are better known as a version of the Bell polynomials $\bar{b}_{n}^{\varphi}(x)[\mathbf{1 5}]$, and can be rewritten [19]

$$
\bar{b}_{n}^{\varphi}(x)=\sum_{k=1}^{n} \sum_{\substack{j_{1}+j_{2}+\cdots=k \\ j_{1}+2 j_{2}+\cdots=n}} \frac{n !}{(1 !)^{j_{1}} j_{1} !(2 !)^{j_{2}}\left(j_{2} !\right) \cdots} \varphi_{1}^{j_{2}} \cdots \varphi_{n-1}^{j_{n}} x^{k} .
$$

So
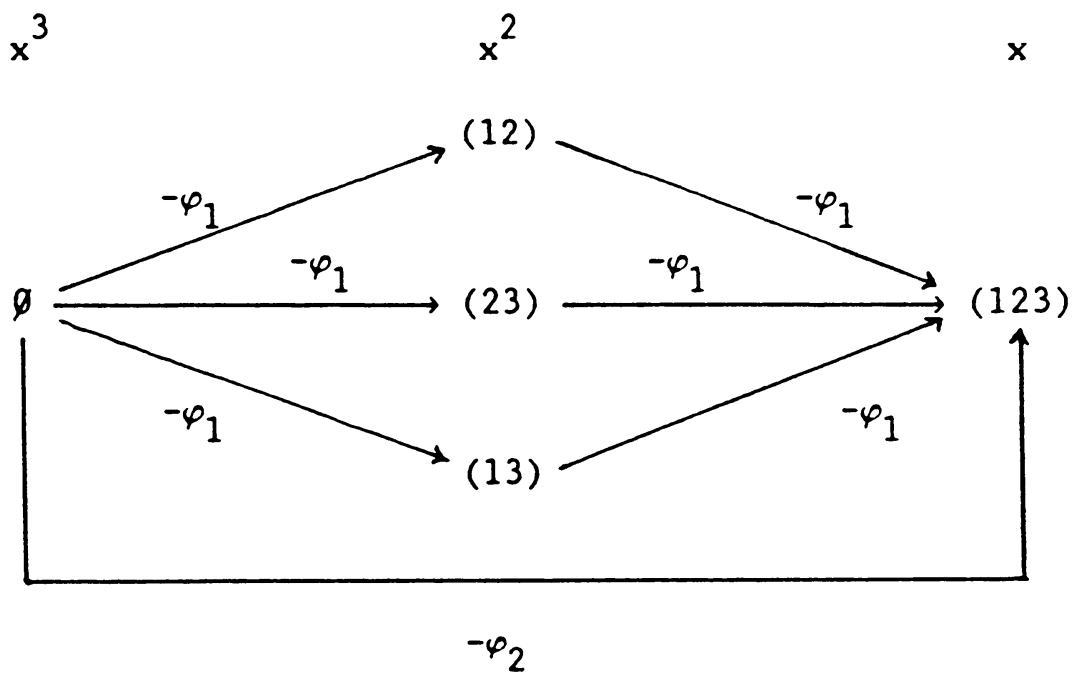

is a diagram for the conjugate Bell polynomial

$$
\begin{aligned}
b_{3}^{\varphi}(x) & =1 \cdot x^{3}+\left(-3 \varphi_{1}\right) x^{2}+\left(3\left(-\varphi_{1}\right)^{2}-\varphi_{2}\right) x \\
& =x^{3}-3 \varphi_{1} x^{2}+\left(3 \varphi_{1}^{2}-\varphi_{2}\right) x .
\end{aligned}
$$

The examples (ii) above collapse to (i) by applying the genus $\gamma$.

As noted in $\S 2$, the role of the $\Delta$-operator in (5.15) is that $\bar{c}_{*}(\omega ; x)$ and $c_{*}(\omega ; x)$ are the conjugate and associated sequences for

$$
\Delta=e^{\omega D}-1, \quad \omega^{i} \equiv \omega_{i-1} .
$$

Since $\omega^{\varphi}(C)$ is universal, so the Bell polynomials (and their conjugates) are the universal binomial sequence, and all others arise by suitable substitution for the $\varphi_{i}$ 's. As we saw in $\S 4$, this is expressed by $\hat{\omega} \bar{b}_{*}^{\varphi}(x)=\bar{c}_{*}(\omega ; x)$ for all $w \in \operatorname{ep}(A)$. If $\omega$ is the Möbius type function $\mu^{\varphi}$, then $\hat{\omega}$ is the automorphism $\bar{b}_{n}^{\varphi}(x) \mapsto b_{n}^{\varphi}(x)$ of $\Phi_{*}[x]$.

The interpretations of $\S 2$ are still valid, in that if $x=|X|$ is an integral variable, we may consider $\bar{c}_{n}(\omega ; x)$ and $c_{n}(\omega ; x)$ as the total weights and Möbius weights respectively of objects in $\omega$ labelled by $X$. However, substituting umbral integers 
for $x$, as in (4.12) produces more subtle results. For example, in the universal case, the characteristic types of $P(\mathbf{n})$ are computed by

$$
\begin{aligned}
b_{n}^{\varphi}(t \varphi) & =c^{\varphi}(\mathcal{P}(\mathbf{n}) ; t \varphi)=\left\langle\left(e^{\varphi D}\right)^{t} \mid b_{n}^{\varphi}(x)\right\rangle \\
& =\left\langle\sum\left(\begin{array}{l}
t \\
j
\end{array}\right)\left(e^{\varphi D}-1\right)^{j} \mid b_{n}^{\varphi}(x)\right\rangle=\left(\begin{array}{c}
t \\
n
\end{array}\right) n ! .
\end{aligned}
$$

Hence

$$
c^{\varphi}(P(\mathbf{n}) ; t \varphi)=[t]_{n} .
$$

We can now use (5.18) to evaluate the expression $(t \varphi)^{n}$ of $(4.12)$, which we need in $§ 6$. From (5.13) and (5.17),

$$
x^{n}=\sum_{\pi \in \mathcal{P}(\mathbf{n})} \varphi_{1}^{\lambda_{2}} \cdots \varphi_{n-1}^{\lambda_{n}} b_{|\pi|}(x),
$$

so

$$
(t \varphi)^{n}=\sum_{\pi} \varphi_{1}^{\lambda_{2}} \cdots \varphi_{n-1}^{\lambda_{n}}[t]_{|\pi|} .
$$

The universal problem also illuminates the Lagrange inversion principle (1.3). Since $e^{\varphi D}-1$ has conjugate sequence $\bar{b}_{*}^{\varphi}(x)$, so $\bar{b}_{n, 1}^{\varphi}=\varphi_{n-1}$. Moreover, by (1.9), $b_{n, 1}^{\varphi}=\bar{b}_{n, 1}^{\bar{\varphi}}=\bar{\varphi}_{n-1}$, so Theorem (5.15) applies.

(5.20) PROPOSITION. Lagrange inversion is a special case of Möbius inversion insofar as, with the above notation, $\bar{\varphi}_{n-1}$ is the Möbius type of 1 in $P(\mathbf{n})$.

For example, (5.17) implies $\bar{\varphi}_{2}=3 \varphi_{1}^{2}-\varphi_{2}$.

Perhaps (5.20) suggests that a simplified combinatorial proof (see [10]) of Lagrange inversion is possible.

6. The bond polynomial of a graph. Our second application of partition categories is to generalise the chromatic polynomial of a graph. Throughout this section we shall draw on Aigner [1] to establish most of our notation and conventions. Thus $G=G(V, S)$ is a graph with vertex set $V$ of $n$ elements, and edge set $S$ containing no loops.

We begin by recalling the bond lattice $\Lambda(G)$ of $G$ (e.g. see [20]), also known as the lattice of flats of the polygon matroid corresponding to $G$. A set of edges $A \subseteq S$ is bond closed if any edge of $G$ not in $A$ has its vertices in distinct connected components of $G(V, A)$, and $\Lambda(G)$ is made up of all such sets, ordered by inclusion.

(6.1) PROPOSITION. The bond lattice $\Lambda(G)$ is the underlying poset of a partition category $\mathcal{L}(G)$.

ProOF. We embed $\Lambda(G)$ as a subposet of $\Pi(V)$, so that $\mathcal{L}(G)$ is defined as the corresponding full subcategory of $P(V)$. Such an embedding is given by assigning to a bond closed set $A$ the partition $\pi_{A}$ of $V$ whose blocks are the vertices of each connected component of $G(V, A)$. It remains only to note that $A \subseteq B \Rightarrow \pi_{A} \leq$ $\pi_{B}$.

(6.2) Definition. We call the characteristic type-polynomial $c^{\varphi}(\mathcal{L}(G) ; x)$ the bond polynomial of the graph $G$, and abbreviate it to $c^{\varphi}(G ; x)$. 
If we take the genus $\gamma$ as weight function, we similarly abbreviate $c(\mathcal{L}(G) ; x)$ to $c(G ; x)$. Of course $\hat{\gamma}: \Phi_{*}[x] \rightarrow \mathbf{Z}[x]$ maps $c^{\varphi}(G ; x)$ to $c(G ; x)$. In fact $c(G ; x)$ is very familiar! $G$.

(6.3) PROPOSITION. The polynomial $c(G ; x)$ is the chromatic polynomial of

PROOF. If $G$ has $k(G)$ components, then the final object of $\mathcal{L}(G)$ has $k(G)$ blocks, so $c(G ; x)$ is the product of $x^{k(G)}$ with the classical characteristic polynomial of $\Lambda(G)$. But this is well known (e.g. see $[\mathbf{1}, 7.54]$ ) to be the chromatic polynomial of $G$.

Thus if we substitute $\varphi_{1}=\cdots=\varphi_{n-1}=1$ in the bond polynomial of $G$, we obtain the chromatic polynomial; it is our promised generalisation.

Recalling Definition (4.13), we may consider the sequences

$$
{ }^{\varphi} G(t)=c^{\varphi}(G ; t \varphi) \quad \text { and } \quad c(G ; t), \quad t=0,1,2, \ldots,
$$

of characteristic types and characteristic weights of $G$, in $\Phi_{*}$ and $\mathbf{Z}$ respectively. Since $\hat{\gamma}\left({ }^{\varphi} G(t)\right)=c(G ; t)$, we deduce from $(6.3)$

(6.4) COROLLARY. If we substitute $\varphi_{1}=\cdots=\varphi_{n-1}=1$ in $\varphi G(t)$, we obtain the number of colourings of $G$ by $t$ colours, $t=0,1,2, \ldots$.

Thus the characteristic types of $G$ incorporate information on the partitions of $V$ effected by colourings of $G$ : we call them bond types after the originating polynomial. Sometimes this information is easy to interpret, but on other occasions is surprisingly obscure. We therefore end with four examples, which illustrate this contradictory behaviour.

(6.5) The complete graph $K_{n}$. Here $\Lambda\left(K_{n}\right)$ is visibly $\Pi(n)$, so that $\mathcal{L}\left(K_{n}\right)$ is $\mathcal{P}(\mathbf{n})$. Hence by (5.16(ii)), the bond polynomial $c^{\varphi}\left(K_{n} ; x\right)$ is the conjugate Bell polynomial $b_{n}^{\varphi}(x)$. So we deduce from (5.18) that the bond types of $K_{n}$ are ${ }^{\varphi} K_{n}(t)=[t]_{n}$, $t=0,1,2, \ldots$

The absence of $\varphi_{1}, \varphi_{2}, \ldots$ from these types suggests that all partitions involved have type $\varphi_{0}^{n}$. This is exactly right, since any of the $[t]_{n}$ colourings of $K_{n}$ can only partition $V$ into singletons.

(6.6) The null graph $N_{n}$. Here $\Lambda\left(N_{n}\right)$ is visibly $0 \in \Pi(n)$, so that $\mathcal{L}\left(K_{n}\right)$ is the initial object and its identity in $P(\mathbf{n})$. Hence the bond polynomial is $x^{n}$, and the bond types of $N_{n}$ are

using (5.19).

$$
\begin{aligned}
\varphi^{N_{n}(t)} & =(t \varphi)^{n}, \quad t=0,1,2, \ldots \\
& =\sum_{\pi \in \mathcal{P}(\mathbf{n})} \varphi_{1}^{\lambda_{2}} \cdots \varphi_{n-1}^{\lambda_{n}}[t]_{|\pi|}
\end{aligned}
$$

Again this is exactly right, since any colouring of the vertices is permissible. So if $k$ distinct colours are used, $0 \leq k \leq n$, they will create a partition $\pi$ of $V$ of type $\varphi_{1}^{\lambda_{2}} \cdots \varphi_{n-1}^{\lambda_{n}}$ with $|\pi|=k$. Permuting the colours gives $[t]_{k}$ choices altogether; so $\varphi^{\varphi} N_{n}(t)$ records all colour partitions of $V$, enumerated by type.

(6.7) The tree T.

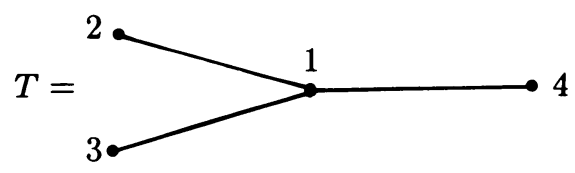


Here $\mathcal{L}(T)$ can be represented as

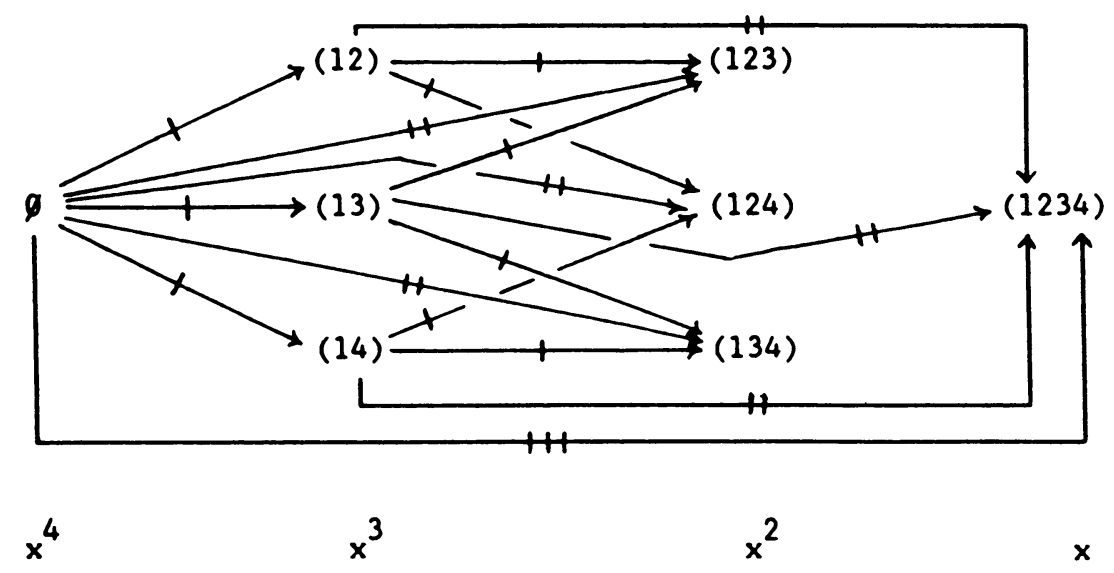

where we have indicated the Möbius type of each $(\pi, \sigma)$ by

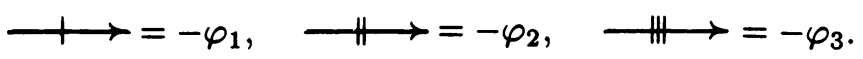

So

$$
c^{\varphi}(T ; x)=x^{4}-3 \varphi_{1} x^{3}+\left(6 \varphi_{1}^{2}-3 \varphi_{2}\right) x^{2}+\left(6 \varphi_{1} \varphi_{2}-6 \varphi_{1}^{3}-\varphi_{3}\right) x
$$

We remark that $\hat{\gamma} c^{\varphi}(T ; x)=x(x-1)^{3}$, which is indeed the chromatic polynomial of $T$, so confirming (6.3).

We now substitute $x=t \varphi$ in (6.8), using (5.19), to obtain bond types

$$
{ }^{\varphi} T(t)=[t]_{4}+3 \varphi_{1}[t]_{3}+\varphi_{2}[t]_{2}, \quad t=0,1,2, \ldots
$$

Again, this exactly records all colour partitions of $T$ by type, as we may indicate by

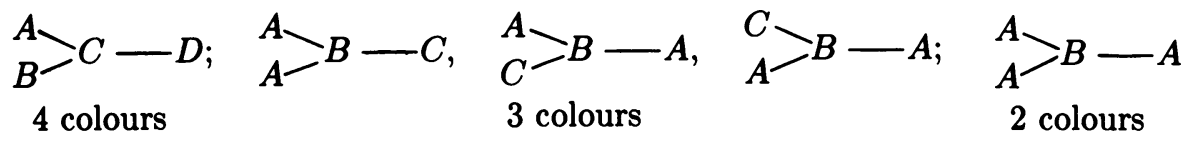

(6.9) The graph $G$.

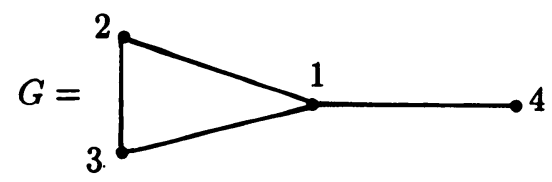


Here $\mathcal{L}(G)$ can be represented as

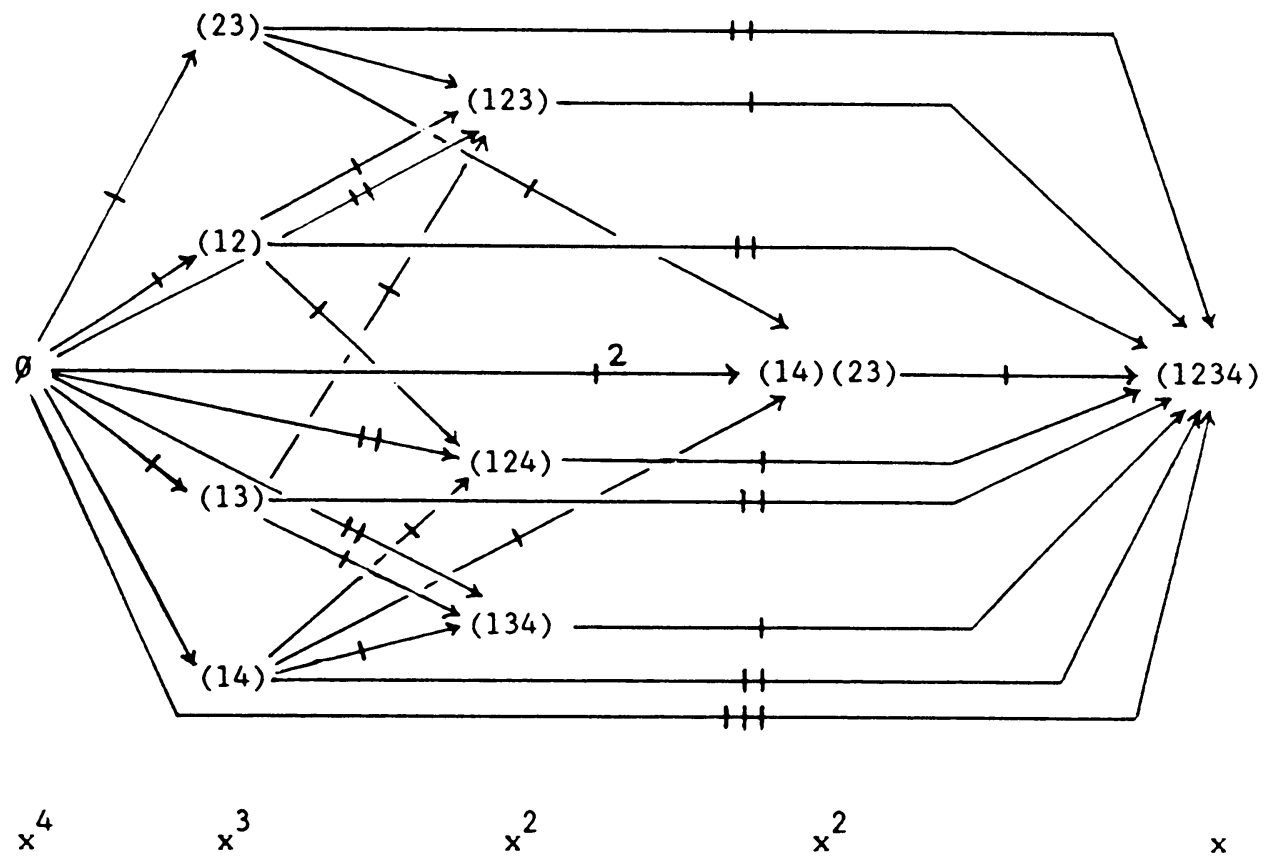

where Möbius types are indicated as in (6.7), plus $\stackrel{2}{\stackrel{2}{\rightarrow}}=-\varphi_{1}^{2}$. So

$$
c^{\varphi}(G ; x)=x^{4}-4 \varphi_{1} x^{3}+\left(8 \varphi_{1}^{2}-3 \varphi_{2}\right) x+\left(7 \varphi_{1} \varphi_{2}-8 \varphi_{1}^{3}-\varphi_{3}\right) x .
$$

We remark that

$$
\hat{\gamma} c^{\varphi}(G ; x)=x(x-1)^{2}(x-2)
$$

which is indeed the chromatic polynomial of $G$, so confirming (6.3).

We now substitute $x=t \varphi$ in (6.10), using (5.19), to obtain bond types

$$
{ }^{\varphi} G(t)=[t]_{4}+2 \varphi_{1}[t]_{3}+\left(\varphi_{2}-\varphi_{1}^{2}\right)[t]_{2} .
$$

In this instance we have failed to describe all colour partitions of $G$ by type, since there are no colourings of $G$ by 2 colours, as is indicated by the fact that $\hat{\gamma}\left(\varphi_{2}-\varphi_{1}^{2}\right)=0$.

This was our main motivation for recent work with C. Wright [17], where we have overcome this defect by defining a new partition category for a graph $G$ whose associated types enumerate the colour partitions of $V$ by type, for all graphs $G$.

However, the bond polynomial still appears to be of some interest; for example it distinguishes between all trees on four vertices, and so is much more powerful than the traditional chromatic polynomial. It would also be satisfying to have a combinatorial interpretation of the information stored in bond types such as (6.11). 


\section{REFERENCES}

1. M. Aigner, Combinatorial theory, Springer-Verlag, 1979.

2. C. Berge, Principles of combinatorics, Academic Press, 1971.

3. J. Blissard, Theory of generic equations, Quart. J. Pure Appl. Math. 4 (1861), 279-305.

4. L. Comtet, Advanced combinatorics, Reidel, 1974.

5. M. Content, F. Lemay and P. Leroux, Catégories de Möbius et fonctorialités; un cadre général pour l'inversion de Möbius, J. Combin. Theory A Ser. 28 (1980), 169-190.

6. P. Hall, A contribution to the theory of groups of prime power order, Proc. London Math. Soc. 36 (1932), 39-95.

7. M. Henle, Binomial enumeration on dissects, Trans. Amer. Math. Soc. 202 (1975), 1-39.

8. S. A. Joni \& G.-C. Rota, Coalgebras and algebras in combinatorics, Stud. Appl. Math. 61 (1979), 93-139.

9. A. Joyal, Une théorie combinatoire des séries formelles, Adv. in Math. 42 (1981), 1-82.

10. G. Labelle, Une nouvelle démonstration combinatoire des formules d'inversion de Lagrange, Adv. in Math. 42 (1981), 217-247.

11. E. K. Lloyd, Enumeration, Handbook of Applicable Mathematics 5, Wiley, 1985, pp. 531-621.

12. S. Mac Lane, Categories for the working mathematician, Springer-Verlag, 1971.

13. R. Mullin and G.-C. Rota, On the foundations of combinatorial theory III: Theory of binomial enumeration, Graph Theory and its Applications, Academic Press, 1971, pp. 168-213.

14. O. Nava \& G.-C. Rota, Plethysm, categories and combinatorics, Adv. in Math. 58 (1985), 61-88.

15. N. Ray, Extensions of umbral calculus: penumbral coalgebras and generalised Bernoulli numbers, Adv. in Math. 61 (1986), 49-100.

16. __ Symbolic calculus: a 19th century approach to $M U$ and BP, Proc. 1985 Durham Sympos. on Homotopy Theory, London Math. Soc. Lecture Note Ser. 117, Cambridge Univ. Press, 1987, pp. $195-238$.

17. N. Ray and C. Wright, Umbral calculus and a new chromatic polynomial, Ars Combinatoria (to appear).

18. J. Riordan, An introduction to combinatorial analysis, Wiley, 1958.

19. S. Roman, The umbral calculus, Academic Press, 1984.

20. G.-C. Rota, On the foundations of combinatorial theory. I: Theory of Möbius functions, Z. Wahrsch. Verw. Gebiete 2 (1964), 340-368.

21. P. J. Webb, A local method in group cohomology, Comment. Math. Helv. 62 (1987), 135-167.

22. E. T. Whittaker and G. N. Watson, A course of modern analysis, Cambridge Univ. Press, 1927.

Department of Mathematics, The University, Manchester, M13 9PL, EngLAND 